\title{
Bilateral Hip Joint Arthritis in a Young Adult
}

\author{
MR SIDDIQUI, ${ }^{1}$ MS MAHBUB, ${ }^{2}$ MAI KHAN ${ }^{3}$
}

\begin{abstract}
A 22 year-old young man presented to us with the complaints of limping gait with recurrent pain in the both hip joints for last 1 year. Recently he also developed a painful swelling of his right upper thigh. On query he gave past history of prolonged bleeding after circumcision at the age of 6 year. Clinical examination revealed a big haematoma in the anterior compartment of his right thigh. He also had features of arthritis of both hip joints. There was marked restriction of medial rotation and of abduction in the both hip joints. Radiographs of the pelvis and both hips showed widening of the joint space and irregularity of the superior-medial articular margin on the both side (fig-1) and marginal sclerosis on the left side (fig-2). He also had prolonged clotting time with a low clotting factor VIII. Finally it was diagnosed as a case of haemophilia with haemophilic arthropathy of the both hip joint.
\end{abstract}

Bleeding into joints and soft tissues are the hallmark of haemophilia. ${ }^{1}$ It is well known that the recurrent haemorrhages into the knee joint of some haemophiliac patients may produce permanent changes in those joints.

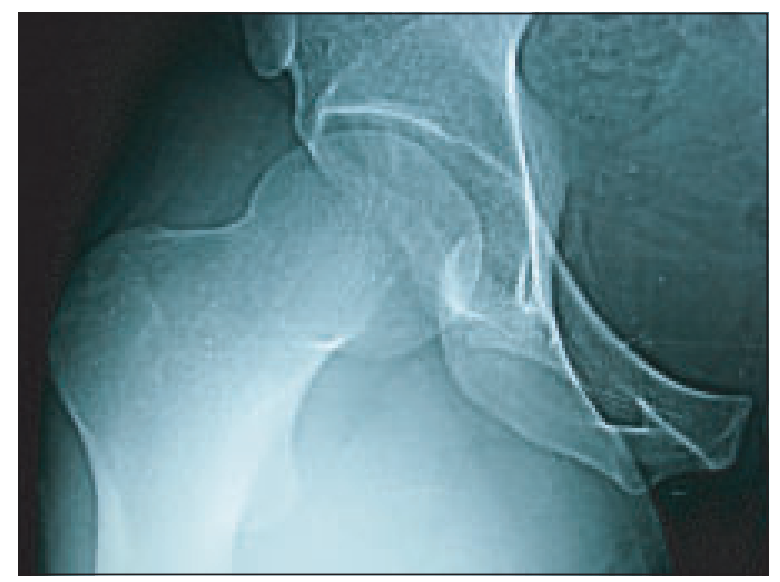

Fig.-1: X-ray of right hip showing widening of the joint space with irregular articular surface.

1. Dr. Mahmudur Rahman Siddiqui, Assistant Professor of Medicine, AKMMC.

2. Dr. Md. Shahriar Mahbub, FCPS P-II Course, DMC

3. Dr. Mohammad Ashik Imran Khan, Medical Officer, 250 Beded Hospiotal Sadar, Gopalgonj,

Correspondence: Dr. Mahmudur Rahman Siddiqui, Assistant Professor of Medicine,AKMMC.E-mail:dr.mahmud99@yahoo.com
But involvement of the both hips is relatively rare. ${ }^{1,2}$ After recurrent haemarthrosis, resorption of intra-articular blood induces reactive synovitis and causes cartilage damage, finally causing complete destruction of it. ${ }^{3}$

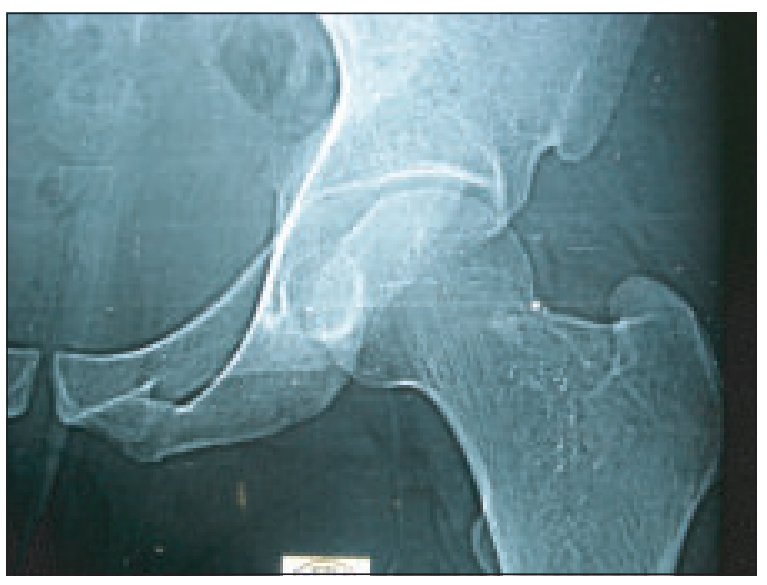

Fig.-2: X-ray of left hip showing widening of the joint space with irregular articular surface \& marginal sclerosis.

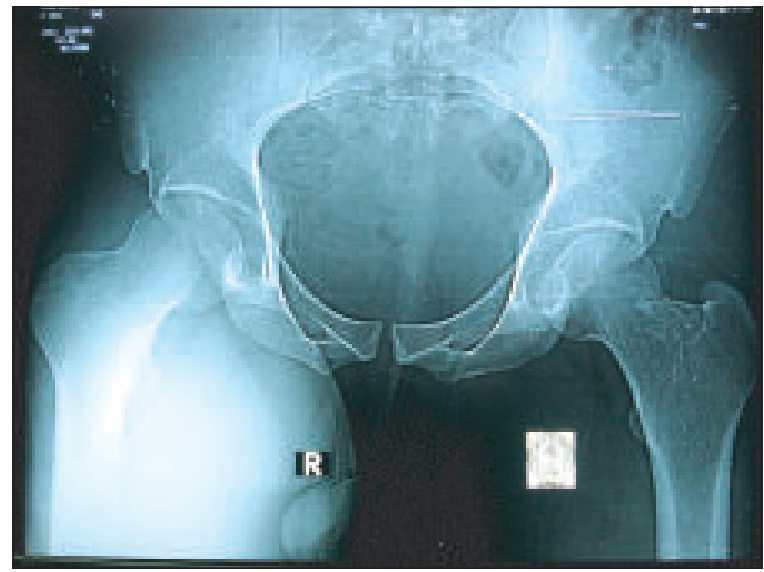

Fig.-3: X-ray of the pelvis showing bilateral hip joint arthropathy with a opacity in right thigh (haematoma).

\section{References:}

1. Mahajan A, Verma S, Singh JB. Haemophilic Arthropathy. JK Science 2005;7(4):228-30.

2. Luck JV Jr, Silva M, Rodriguez-Merchar EC et al. Haemophilic arthropathy. J Am Acad Orthop Surg 2004; 12(4): 234-45.

3. Knobe K, Berntorp E. Haemophilia and joint disease: pathophysiology, evaluation, and management. Journal of Comorbidity 2011;1:51-59. 